\title{
How to enhance experience and skill of non invasive ventilation: suggestions from the literature
}

\author{
Antonello Nicolini ${ }^{1}$ \\ Ines Maria Grazia Piroddi ${ }^{1}$ \\ Cornelius Barlascini²
}

\author{
${ }^{1}$ Respiratory Diseases Unit, General Hospital of Se- \\ stri Levante, Sestri Levante (GE), Italy \\ 2 Hygiene and Health Medicine, Hospital of Sestri Le- \\ vante, Sestri Levante (GE), Italy
}

\author{
Address for correspondence: \\ Antonello Nicolini, MD \\ Respiratory Diseases Unit \\ General Hospital of Sestri Levante \\ Via Terzi 43 - 16039 Sestri Levante (GE), Italy \\ Phone: +390185329145 - Fax: +390185329121 \\ E-mail: antonellonicolini@gmail.com
}

\section{Summary}

The literature relevant to non invasive ventilation (NIV) synthesizes the most recent trends in enhancing experience and skill of NIV team care.

Studies concerning ventilation team-based practices but also single respiratory health professional education and training were included in our research.

Critical care education and training and inter-professional education should start in medical and post graduated school. Curriculum design, implementation, and assessment of clinical skills completed by the trained medical expert are essential components of competency-based education.

Nurses, respiratory therapists and physiotherapists have different roles in ventilation team care around the world. Delivering effective training for NIV is challenging. Team-based care using patient simulation education as an adjunct to traditional clinical training (patient care, didactics, small group teaching and protocols) is superior to traditional clinical training alone and can affect positively patient and provider outcomes.

KEY WORDS: non invasive ventilation (NIV), ventilation team skill, critical care, patient simulation, education and training.

A historical view on non invasive ventilation (NIV)

Most people under the age of 40 are unlikely ever to have seen an iron lung, yet sixty years ago these medical mechanical monsters would have been a common sight in most hospital throughout the world. Through the late 1920's and into 50's the iron lung was considered to be state of the art, high-tech,

life support technology. Indeed medical physician of the time would have learn about such devices as a recommended treatment for respiratory paralysis, used to maintain life for those whose breathing capabilities had been impaired or destroyed by poliomyelitis (1). They
An emerging aspect has been the demand for an increase in collaborative care and ventilation team education in order to deal with the complexity of NIV application.

\section{were non invasive in the sense}

that no part of the device penetrated the patient. In truth, it was the patient who was inside the machine. The whole body was enclosed within the air tight chamber of the device, apart from the head which protruded through a tight seal around the neck (Figure 1). For each inspiration a large set of leather bellows mounted in a separate pump unit would expand causing the pressure within the cabinet to be lowered to below that of the surrounding atmosphere.

The sub-atmospheric pressure in turn acted upon the chest, causing it to expand, thereby drawing fresh air into the lungs through the patients' mouth.

During expiration, the pressure equalise to atmospheric and the patient exhals passively. This method of artificial respiration was known as external negative pressure ventilation (ENPV). The first description of ventilator support via mouthpiece was described by Dr.Affeldt of Rancho Los Amigos in 1953 (Figure 2), to assist patients when the iron lung was open for patient care (1, 2). He observed that intermittent positive pressure administered by a circuit with a mouthpiece could be used to relieve dyspnea in patients with polio ventilator-dependence when negative pressure ventilation had been interrupted for transfers, nursing or physical therapy $(1,2)$. However, intermittent positive pressure breathing (IPPB) machine became available even earlier than 1951 and a positive pressure blower caller was used to deliver occasional deep breaths as well. Patients used mouthpiece NIV in a wheelchair as well as use an intermittent abdominal pressure ventilator (IAPV) or other portable negative pressure ventilators. Since IPPV via mouthpiece Bantam became available and since the IP. $\mathrm{PV}$ via mouthpiece was able to provides much larger volumes of air directly to the lungs than the IAPV, it became the principal mode of ventilatory support during lung infections and other conditions of lung impairment. The mouthpieces were mounted on the controls of powered 


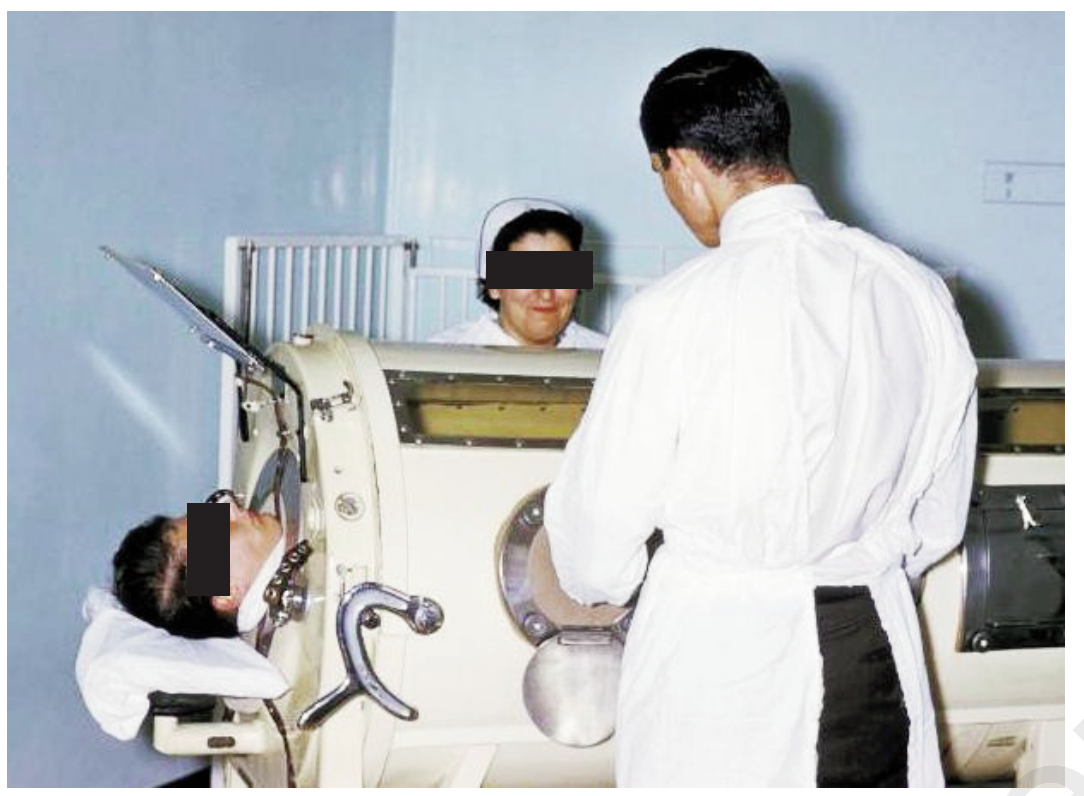

Figure 1 - Staff in a Rhode Island hospital are examining a patient in an iron lung examined by the medical staff, Rhode Island Hospital, Providence, RI (1960).

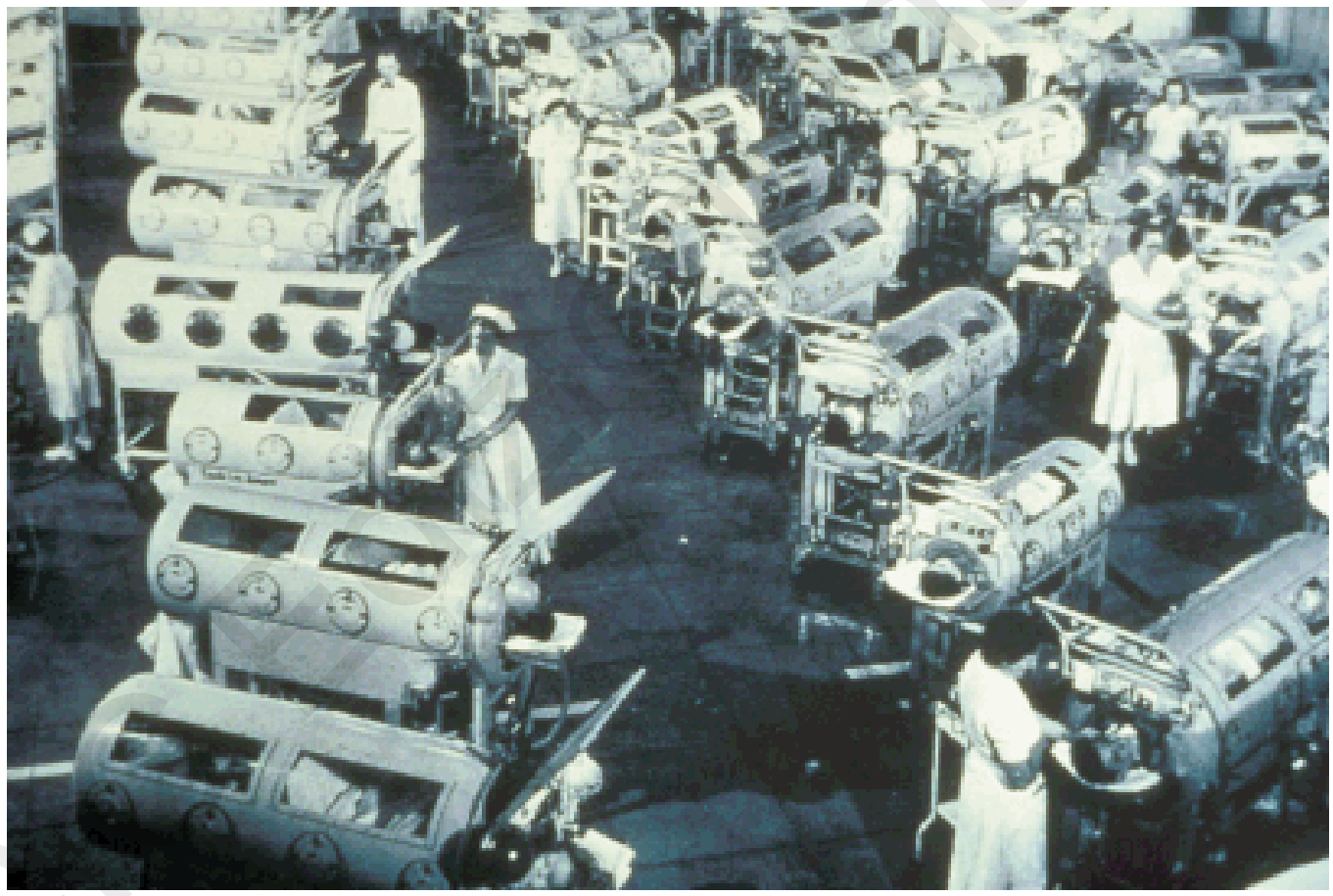

Figure 2 - Iron lung ward filled with polio patients, Rancho Los Amigos Hospital, California (1953).

wheelchairs (with breath control triggers: touch, chin, tongue) adjacent to the mouth for easy access by the patient. Thus, intermittent positive pressure ventilation via a mouthpiece (Figure 3) was the first kind of NIV support, a tremendous advance (which avoided undergoing tracheostomy) in ventilator-dependent patients (1, 3). NIV is a technique that may be not tolerated above all if the interface is uncomfortable. Fortunately over the time we have seen an amazing development of mechan- ical ventilators and increasing number of masks or in terfaces to choose from (4).

The use of NIV varies greatly between hospitals and geographical regions and has changed over the time. Investigators of a worldwide prospective survey of mechanical ventilation noted that use rose from $4 \%$ of all ventilators started in 2001 to $11 \%$ in $2004(5,6)$. It is increasingly being used in many countries, but frequency of use is variable (7). In Europe the rate of use of NIV 


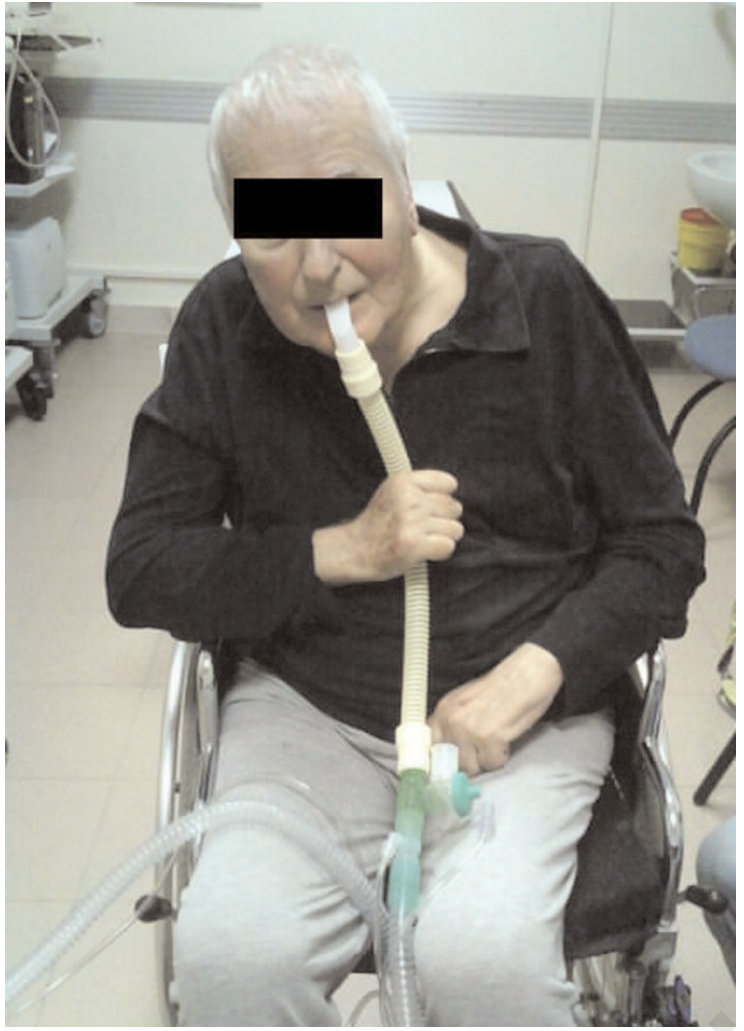

Figure 3 - Open circuit mouthpiece ventilation.

in intensive care is about $35 \%$ of ventilated patients and higher (about 60\%) in respiratory intensive care units (RICUs) or emergency departments $(8,9)$. In North America this form of ventilation is started most often in emergency department, with most patients transferred to intensive care units or step-down units in hospitals that have such facilities. The low rate of using NIV in some hospital may be due to poor knowledge or experience about the technique, insufficient technical equipment and inadequate funding $(6,9)$.

Despite these limitations, NIV is increasingly being used outside ICU: respiratory care units, emergency departments, post surgical recovery rooms, intensive cardiology units, stroke units, oncology wards, and palliative care units (6). An emerging aspect has been the demand for an increase in collaborative care and ventilation team education in order to deal with the complexity of NIV application (6).

For inter-professional teams of healthcare providers this means working collaboratively in the provision of integrated and continuous healthcare.

Team-based healthcare and practice has been widely argued to provide not only improved effectiveness, efficiency and quality of service, but also inappropriate variations in NIV practice.

\section{Review of the literature}

We focused on the small number of recently published studies in order to capture the most recent trends in en- hancing experience and skill of NIV team care and to build upon the existing reviews reported in the literature. This literature review includes reports of qualitative, quantitative and mixed method educational intervention from inter-professional education of health sciences students to simulation-based education for clinicians in respiratory critical care team.

Studies were required to inadept at selecting patients who are likely to succeed with NIV and promptly intubating patients likely to fail NIV.
Physicians need to be

clude one or more of the following criteria: (1) ventilation team skills, (2) NIV educational program or interventions, (3) documented educational and training experiences of ventilation teams, (4) critical care, (5) simulation education.

The following databases were searched: Cochrane Database of Systematic Reviews, MEDLINE, Biomed Central, PubMed.

\section{The key component of NIV success: a skilled and ex- perienced team}

According to the recent literature, the experience and skill of the personnel who manage NIV are the key components of NIV success. Over an 8-year span in an
Curriculum design, implementation, and assessment of clinical skills completed by the trained medical expert are essentials components of competencybased education. Italian ICU, Carlucci et al. (10) found that their overall NIV success rate remained steady despite an increasing severity of illness of patients treated with NIV and concluded that increasing experience with NIV over time allowed caring for sicker patients while maintaining the same success rate. Over a 7-year period in a French ICU, Girou et al. (11) found that the utilization rate of NIV among almost 500 patients who presented with acute respiratory failure (ARF) due to either chronic obstructive pulmonary disease (COPD) or congestive heart failure rose from approximately $20 \%$ to over $80 \%$ of initial ventilator starts. During that same period, the rate of ventilator-associated pneumonia dropped from $20 \%$ to $8 \%$, with a corresponding drop in mortality.

Girou et al. (11) attributed the improved outcomes to
Improving the education and training of practitioners in NIV should be a key part of any measures desiutilization rates and enhance the success of NIV therapy. gned to increase NIV routine use of NIV and a

"learning effect." Although difficult to be proved, both Carlucci et al. and Girou et al. studies $(10,11)$ attributed outcomes improvements to increasing experience and skill of the staff.

Optimal management of NIV requires that all members of the team be experienced and skillful. Physicians need to be adept at selecting patients who are likely to 
succeed with NIV and promptly intubating patients likely to fail NIV. In North America, Respiratory Therapists (RTs) are the most often responsible for applying NIV. They must be skilled at selecting an appropriate mask, fitting it to optimize comfort, and adjusting the ventilator to efficiently alleviate respiratory distress. Nurses need to be knowledgeable about monitoring, to help avoid and detect problems. Quantifying the experience and skill of a unit's staff is challenging because individuals differ considerably and personnel changes can have important effects. However, as units use more NIV, outcomes appear to improve. Periodic in-services and updates probably help personnel maintain their skills and stay abreast of new developments (12).

\section{Medical education and training}

Medical students should be given some form of early clinical education in NIV.

Working directly with Emergency Medical Technicians (EMTs), student confidence in patient care skills showed a significant increase from baseline for all identified skills. Student confidence in team-building skills showed a significant increase. Moreover, a concurrent EMT course builds confidence. By the end of the first year, $84 \%$ of the first year students reported the EMT curriculum had 'some impact' to 'great impact' on their patient care skills, while $72 \%$ reported that EMT curriculum had 'some impact' to 'great impact' on their team-building skills. The incorporation of EMT training early in a medical school curriculum provides students with meaningful clinical experiences that increase their self-reported level of confidence in the performance of patient care skills early in their medical education (13).

Inter-professional education (IPE) is an approach that encourages collaboration between health care professionals, optimizing health care quality and decisionmaking processes (14).

Effective communication across multiple healthcare disciplines and professions is critical to ensure the delivery of effective care $(15,16)$. Yet, many healthcare professionals enter practice without sufficient training in inter-professional care and coordination (17). IPE transcends mono-professional and isolated approaches to health personnel education. It emphasizes interactive learning with and from members of other professions to improve professional practice and care delivery (17). Mitchell et al. (18) has reported that "Effectiveness of learning is enhanced when a mentor supports the portfolio process". Within the competency-based framework, education is no longer a teacher-driven; the learner is expected to share this responsibility. However, the medical teacher has assumed additional responsibilities involved in facilitating the learning cycle, and contribution to curriculum development.

Curriculum design, implementation, and assessment of clinical skills completed by the trained medical expert are essential components of competency-based education. Placed within the protocols of the various European Respiratory Society (ERS) are educational programs which mandate direct observation of procedural skills and learner self-directed assessment: the Harmonized Education in Respiratory Medicine for European Specialists (HERMES) project $(18,19)$.

The challenge for the HERMES initiative is to strike a balance between expected outcomes for the allied health professional and what the allied health professional is legally permitted to perform within individual countries. Further consideration must also be given to an increased focus on the treatment and diagnosis of disease across specialties. This reality leads to the possibility of team-based learning in delivery of the curricula (18)

The ultimate aim of HERMES is to ensure that the best quality of care is delivered through harmonized educational standards in the context of evolving medical and economic demands.

The curriculum therefore defines specific competencies and provides a usable framework in the training of clinicians in respiratory critical care and aspires to set the stage for consolidation of an additional qualification and competence in respiratory critical and home care medicine.

In this setting, the ERS can hopefully participate in a multi-society effort to improve and facilitate the homogenization and standardization of training across Europe, and the creation of uniform standards of care for critically ill respiratory disease patients (19).

\section{Nurses and Physiotherapists education and train- ing}

According to the American Journal of Critical Care, the role and the scope of practice of acute care nurse practitioners (ACNPs) continue to expand.

Affecting patients' outcomes was identified as an advantage of being an ACNP.

Participants report that they are making a fairly high impact on outcomes, including length of stay, costs, readmission rates, adherence to best practices, medical management, complications, resource utilization, continuity of care, patients' access to care, patients' satisfaction, and education of patients, patients' family members, and staff. As the results of this 5-year national study reflect, the practice settings of ACNPs are diverse. By virtue of educational preparation and national certification, ACNPs focus their care on patients with acute and critical illnesses (19).

In the United States, RTs usually manage the NIV/CPAP adaptation in emergency or respiratory clinics and subsequent execution of respiratory care therapeutic treatments (Figure 4).

In Europe, RT is not a recognized profession, while physiotherapists (PTs) who have additional specialized training in respiratory care are usually involved in respiratory departments. However, there may be important differences in tasks and responsibilities among different countries and hospitals.

European surveys have demonstrated the feasibility of implementing protocols that include PTs as an integral part of a cardiopulmonary rehabilitation service (CPRS) team. 


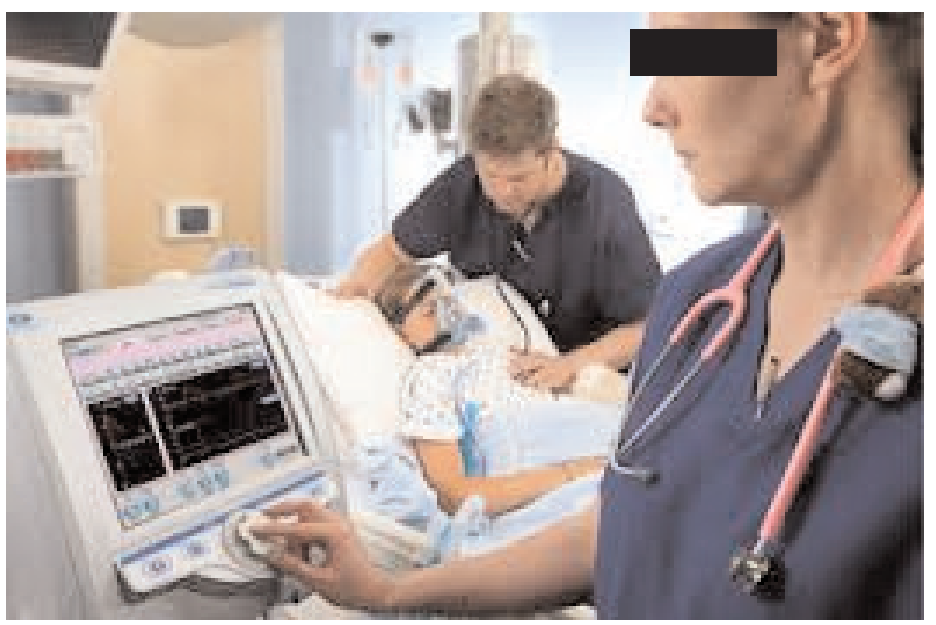

Figure 4 - NIV setting into the hospital is usually driven by respiratory therapists (RTs) in North America.

In Italy, the assessment, education, and care for patients with pulmonary disorders, in acute and chronic settings, are managed mainly by pulmonologists and nurses. PTs may be involved as part of the dedicated care team in tasks related to monitoring a patient's physiological responses to pharmacologic therapies (e.g. vital signs, pulse-oximetry), to supervise exercise training sessions, and to perform airways clearance techniques (20).

The effect of the introduction of RTs in NIV management teams has been examined in only one study, by Kareus et al. (21). These authors demonstrated an improvement in NIV tolerance after RT involvement, increasing patient tolerance to NIV by about $51 \%$.Nava (22) reports that in Europe, NIV seems to be more popular than elsewhere, perhaps because the first randomized controlled trials were performed in France and the United Kingdom. It may be more popular there also because the physician has the direct responsibility in ordering and applying NIV with the support of all the staff, including nurses and RTs, while in most other continents the prescription is basically ordered by the physician but is independently applied by the RT; this might affect the teamwork (22)

\section{Ventilation team training}

The low rate of NIV use in some hospitals relates to lack of knowledge and experience with NIV, insufficient confidence in the technique, lack of NIV equipment, and inadequate funding. But NIV use has been increasing around the world, thanks partly to improved technologies. The skill and confidence of clinicians in NIV have improved with time and experience, but NIV is and should remain a team effort (22).

Protocols are used as a strategy to lower the occurrence of medical errors, omissions, and delays. They may reduce conflicts between team members, improve outcomes, decrease costs, and increase educational efforts.

The development of protocols and guidelines for
NIV/CPAP management is recommended in order to clarify the roles of each professional and to increase greater efficiency during NIV application (20).

An example of how adequate risk assessment tools/protocols may help nurses considerably in enhancing patient safety is The Burns Wean Assessment Program checklist, developed to assist clinicians in the systematic evaluation of 26 clinical factors important to weaning (23).

Good inter-professional communication influences quality of care and patient safety. Regular ward rounds with documented outcomes and review of defined treatment goals between nursing, and medical staff have been shown to reduce length of ICU stay. The gold standard teaching tool is individual feedback on videos of real meetings (24).

This can be extended to an assessment tool where a portfolio of consultations is submitted for review by an examiner using agreed criteria.

The practical and ethical difficulties of this technique have been overcome in primary care and there is an opportunity for critical care medicine to lead other hospital specialties in this area (24).

Competency in communication is a core clinical skill, which must be taught, tested, and practiced. An understanding of the evidence and theory of communication coupled with a commitment to cultivate and improve skills not only benefits patients and their families, it can also invigorate professional practice and provide an ongoing clinical challenge $(24,25)$.

It is nevertheless interesting to note that according to Carlucci et al. (10) introduction of a clinical diary for the monitoring and collection of vital signs and ventilatory settings may improved and standardized the approach to NIV by paramedics. All the nurses working inside and outside the Respiratory ICU underwent educational courses twice a year, which included the practice of NIV delivery, cardiopulmonary resuscitation, and the management of life-threatening emergencies. The RTs were also progressively taught the basics of mechanical ventilation, and the practice of NIV delivery in the acute setting. 


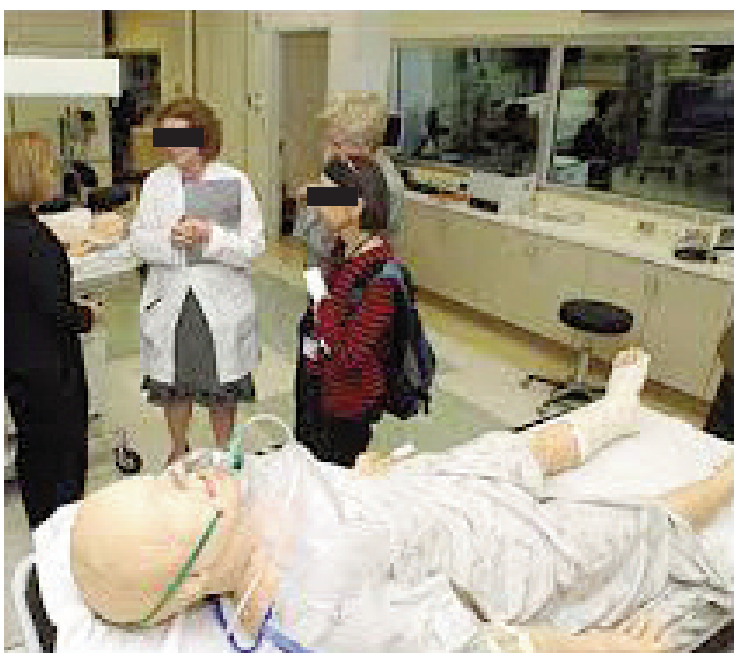

Figure 5 - Clinical simulation-based medical education with mannequin at Vanderbilt University School of Medicine, Nashville, TN.

Both medical and paramedical personnel in their study participated in courses outside the hospital and attended major national and international meetings; the number of publications also progressively increased, so that the motivational aspect of the medical and paramedical staff also may have improved.

Improving the education and training of practitioners in NIV should be a key part of any measures designed to increase NIV utilization rates and enhance the success of NIV therapy. Conferences and lectures often have little effect in terms of changing clinical practice, and practical hands-on experience is more consistently successful. Medical simulation is ideally suited to this hands-on training and has previously been shown to be effective in training nursing staff in the practical use of NIV (Figure 5).

The level of training and immersion can be increased by creating a realistic working environment such as a simulated ICU or operating room coupled with feedback from observers and video cameras to assist improvement in skills $(25,26)$. Clara et al. also show that simulation-based education used as an adjunct to the traditional clinical training (patient care, didactics, and small group teaching) is superior to traditional clinical training alone. Simulation-based education is a feasible intervention that improves the educational experience and boosts clinical knowledge and skill (26).

\section{Conclusions}

Clinical expertise with NIV is the most important factor in its successful use $(22,28-32)$. A systematic review of the of the predictors of NIV failure found that patient tolerance and compliance with NIV is closely related to clinician expertise (29): this has been confirmed by other studies $(10,11,27,28,30-32)$. Taken together these studies suggest that improving education and training of NIV team should be a key part of any program de- signed to increase NIV utilization rates and enhance NIV success.

Delivering effective training for NIV is challenging. A simulation-based educational tool for NIV proposed by Hare A. (25) addressed to clinicians and other hospital personnel involved in the delivery NIV should be considered as an ideal example of continuous educational activity effective in terms of clinical skills acquisition and translation. Continuous self and inter-team training is another method to enhance the skills and the performances in respiratory critical care. However this preparation takes time. The authors believe this time as an investment that will save lives as well as medical and economic resources.

\section{References}

1. Bach JR, Gonclaves MR, Hon A, Ishikawa Y, De Vito $\mathrm{EL}$, Prado F, Dominguez ME. Changing trends in the management of end-stage neuromuscular respiratory muscle failure. Am J Phys Rehabil. 2012;91:1-11.

2. Bach JR, Alba AS, Bohatiuk G, Saporito L, Lee M. Mouth intermittent positive pressure ventilation in the management of post polio respiratory insufficiency. Chest. 1987;91:859-864.

3. Garuti G, Nicolini A, Grecchi B, Lusuardi M, Winck JC, Bach JR. Open circuit mouthpiece ventilation: concise clinical review. Port J Pulm. 2014;20:211218.

4. Hess DR. The growing role of non invasive ventilation in patients requiring prolonged mechanical ventilation. Respir Care. 2012;57:900-918.

5. Esteban A, Ferguson ND, Meade MO, et al. Evolution of mechanical ventilation in response to clinical research. Am J Resp Crit care Med. 2008; 177:170-177.

6. Nava S, Hill N. Non-invasive ventilation in acute respiratory failure. Lancet. 2009;374:250-259.

7. Crimi C, Noto A, Esquinas A, Nava S. Non-invasive ventilation (NIV) practices: a European web-survey. Eur Resp J. 2008;32:1747-1755.

8. Vanpee J, Delaunois L, Lhereux P, et al. Survey of non-invasive ventilation (NPPV) in patients with acute exacerbations of chronic obstructive pulmonary disease patients in emergency department in Belgium. Eur J emerg Med. 2002;9:217-224.

9. Maheshwari V, Paioli D, Rothaar R, Hill N. Utilization of noninvasive ventilation in acute care hospitals: a regional survey. Chest. 2006;129:1226-1233.

10. Carlucci A, Delmastro M, Rubini F, Fracchia C, Nava $S$. Changes in the practice of non-invasive ventilation in treating COPD patients over 8 years. Intensive Care Med. 2003;29(3):419-425.

11. Girou E, Brun-Buisson C, Taillé S, Lemaire F, Brochard L. Secular trends in nosocomial infec- 
tions and mortality associated with noninvasive ventilation in patients with exacerbation of COPD and pulmonary edema. JAMA. 2003;290(22):29852991.

12. Hill NS. Where should Noninvasive Ventilation be delivered? Respir Care. 2009;54:62-69.

13. Kwiatkowski T, Rennie W, Fornari A, Akbar S. Medical students as EMTs: skill building, confidence and professional formation. Med Educ Online. 2014,19:24 829.

14. Melche P, Meyenberg C, Douchamps L, Theubet A, Emilien J. Design and implementation of an interprofessional education course for undergraduate students at the University of Applied Sciences Western Switzerland: the Geneva experience. J Interprof Care. 2014, Early Online: 1-2.

15. Abu-Rish E, Craddick E, Chen LL, Kim S, Blondon $\mathrm{K}$, Rich $\mathrm{J}$ et al. Current trends in interprofessional education of health sciences students: A literature review. Journal of Interprofessional Care. 2012;26: 444-451.

16. Greiner AC, Knebel E. (Eds.). Health professions education: A bridge to quality. Committee on the Health Professions Education Summit. 2003. ISBN: 0-309-51578-1.

17. Barr H. Interprofessional education: Today, yesterday, and tomorrow. The Learning and Teaching Support Network for Health Sciences \& Practice from the UK Centre for the Advancement of Interprofessional Education. 2002.

18. Mitchell S, Steenbruggen I, Paton J, Simonds AK, Bloch KE, Hare A, et al. "Education is the passport to the future": enabling today's medical teachers to prepare tomorrow's respiratory health practitioners. Eur Respir J. 2014;44:578-584.

19. Artigas A, Dellweg D, Nava S, Pelosi P, Rohde G, Schoenhofer B, et al. Defining a training framework for clinicians in respiratory critical care. Eur Respir J. 2014;44:572-577.

20. Kleinpell RM. Acute care nurse practitioner practice: results of a 5-year longitudinal study. Am J Crit Care. 2005;14:211-219.

21. Kareus SA, Kagebein S, Rudnicki SA. The importance of a respiratory therapist in the ALS clinic.
Amyotroph Lateral Scler. 2008;9:173-176.

22. Nava S. Behind a Mask: Tricks, Pitfalls, and Prejudices for Noninvasive Ventilation. Respir Care. 2013:58:1367-1376.

23. Danckers M, Grosu H, Jean R, Cruz RB, Fidellaga $A$, Han $Q$, et al. Nurse-driven, protocol-directed weaning from mechanical ventilation improves clinical outcomes and is well accepted by intensive care unit physicians. J Crit Care. 2013;28:433-441.

24. Gauntlett RT, Laws D. Communication skills in critical care. Continuing Edu Anaesth Crit Care Pain. 2008;8:121-124.

25. Hare A, Simonds A. Simulation-based education for non-invasive ventilation. Breathe. 2013;9:367-371.

26. Rashmi Datta C, Upadhyay KK, Jaideep CN. Simulation and its role in medical education. MJAFI. 2012;68:167-172.

27. Schroedl CJ, Corbridge TC, Cohen ER, Fakhran SS, McGaghie WC, Wayne DB. Use of simulationbased education to improve resident learning and patient care in the medical intensive care unit: A randomized trial. Journal of Critical Care. 2012;27:219. e7-219.e13.

28. Nicolini A, Ferrera L, Santo M, Ferrari-Bravo M, Del Forno M, Sclifò F. Noninvasive ventilation for hypercapnic exacerbation of chronic obstructive pulmonary disease : factors related to noninvasive ventilation failure. Pol Arch Med Wewn. 2014; 124:525-531.

29. Ozyilmar E, Ozsancak U, Nava S. Timing of noninvasive ventilation failure: causes, risk factors, and potential remedies. BMC Pulm Med. 2014; 14:19.

30. Nava S, Ceriana P. Causes of failure of noninvasive mechanical ventilation. Respir Care. 2004;49:295303.

31. Contou D, Fragnoli C, Cordoba-Izquierdo A, et al. Noninvasive ventilation for acute hypercapnic respiratory failure: intubation rate in an experienced unit. Respir Care. 2013;58:2045-52.

32. Burns KE, Sinuff T, Adhikari NK, et al. Bilevel noninvasive positive pressure ventilation for acute respiratory failure : survey of Ontario practice. Crit Care Med. 2005;33:1477-1483. 